\title{
MATHEMATICAL ANALYSIS OF FRACTIONAL ORDER CO-INFECTION TB AND HIV MODEL
}

\section{MUHAMMAD FARMAN, MUHAMMAD USMAN, AQEEL AHMAD*, M.O. AHMAD}

Department of Mathematics and Statistics, The University of Lahore, Lahore, Pakistan

*Corresponding author: aqeelahmad.740@gmmail.com

\begin{abstract}
A mathematical model of HIV/AIDS and TB including its co-infections is formulated. We find the Equilibrium points and with the help of numerical simulation, we have analyzed that the sub-models of TB, HIV/AIDS and its co-infections. The Caputo and Caputo Febrizo fractional derivative operator of order $\alpha \in(0,1]$ is employed to obtain the system of fractional differential equations. Laplace Adomian Decomposition Method was successfully used for solving the different differential equations.Laplace transform is a perfect technique in various field of biological science,engineering,pure and applied mathematics. The latest technique Laplace Adomian Decomposition Method is employed on the developed fractional order model for the numerical solutions. Finally numerical simulations are also established to investigate the influence of the system parameter on the spread of disease and which show effect of fractional parameter $\alpha$ on our obtained solutions.
\end{abstract}

\section{INTRODUCTION}

Mathematics plays a key role in many scientific disciplines, primarily as a mathematical modeling tool. Mathematics may be thought to play an equally important or even less important role in biology, and mathematics and biology may have something in common. Although physics and biology are very different sciences, mathematicians and biologists have developed "mathematical biology" or "biomathematics" in the latest disciplines of biological mathematical characterization, as well as research in biology, biomedical and

Received 2019-10-29; accepted 2019-11-21; published 2020-01-02.

2010 Mathematics Subject Classification. 37C75, 65L07.

Key words and phrases. dynamical transmission; Caputo fractional derivative; simulation; LADM.

(C)2020 Authors retain the copyrights of their papers, and all open access articles are distributed under the terms of the Creative Commons Attribution License. 
biotechnological sciences. The emphasis on biomathematics by the current departmental name is undoubt-

edly a response to the rapid growth in the use of mathematics in biology in recent years [1], but it is in line with my official duties, including the explicit mention of statistics. The mathematician has taken initiatives, but there is no precedent. As Prof. EJ Williams of Melbourne stated in his review of the development of Biomathematical models [2], a mathematical model of any phenomenon can only contribute to understanding when it comes to conclusions that can be tested at using experimental or experimental evidence. Operational test theory is nothing more than pure mathematics, even though the subject of the discussion is described in biological terms.

The use of mathematics in biology is important because biology becomes more quantitative. This new discipline has applications in the fields of biology, biomedicine and biotechnology. The goal of this new discipline is to mathematically represent and model biological problems using various theories and mathematical techniques. Mathematical biology has dramatic and practical applications in the fields of biology, biomedicine and biotechnology. The long-term interaction between mathematical methods and biology is explained by the problematic approach: how does the brain work? Mathematics plays a role in brain research, from Von Helmholtz's [3] early work, which looks for functions similar to energy, the physical and chemical foundations that describe brain dynamics, and Freud's catharsis [4]. Norbert Wiener's cybernetics to study biological control mechanisms [5], mathematical research on the nervous system [6] and recent use of mathematics to study consciousness [7,8]. Examine the Dynamical transmission and effect of smoking in society [15]. The distinguished features of fractional differential equations are that it outlines memory and transmitted properties of numerous mathematical models. As a fact, that fractional order models are more realistic and practical than the classical integer order models. Fractional order derivative produces greater degree of freedom in these models. Arbitrary order derivatives are powerful tools for the discretion of the dynamical behavior of various biomaterial and systems $[16,17]$.

Tuberculosis and AIDS Tuberculosis and HIV co-infection refer to people with both HIV infection and latent or active TB. When a person has HIV and TB, each disease accelerates the progression of another disease. In addition to the accelerated progression of HIV infection from latent to active TB, tuberculosis has accelerated the progression of HIV infection [9]. HIV infection and TB bacterial infections are completely different infections. If you are infected with HIV, you will not be infected with TB unless you are in contact with someone who is infected with TB. However, if you live in a country where the prevalence of TB is high, it can happen if you do not realize it. Similarly, if you have TB, you will not be infected with HIV unless you engage in activities such as unprotected sex with people already infected with HIV. Tuberculosis also occurs earlier than many other opportunistic infections during HIV infection. Co-infected people are twice as likely to die as HIV infected people without TB. This is true even when considering antiretroviral treatment (ARV) [10]. 
The World Health Organization (WHO) estimates that between 2005 and 2012, cooperation activities in tuberculosis prevention and treatment, including TB prevention, antiretroviral therapy and HIV testing, were reinstated at 1.3 million. As a result, these cooperative activities have infected humans by reducing tuberculosis and HIV. In recent years, the number of TB-related deaths among people living with HIV has decreased [11]. Overall, the lifetime risk of HIV-negative patients from latent progression to active progression is estimated at about 5-10 percent. For HIV-positive people, the same number represents a 2-year risk [12]. In 2016, approximately 374,000 people with TB and HIV were killed. This is a supplement to the death of only 1.3 million people with TB [13]. It has been reported that people co-infected with HIV and tuberculosis die as a result of HIV infection [14]. The symptoms of active TB and AIDS make you feel sick. The cough lasts more than 2 weeks, coughing mucus or blood, chest pain. You may also have weakness or fatigue, weight loss, lack of appetite, fever or chills, and night sweats.

The link between HIV and TB is opportunistic infection (OI). For people whose immune systems are weakened, infections occur more often or more severely than those with healthy immune systems. Treatment with anti-HIV drugs is called antiretroviral therapy (ART). Antiretroviral therapy protects the immune system and prevents HIV infection from contracting AIDS. In people co-infected with HIV and TB, antiviral therapy may reduce the risk of developing latent tuberculosis tuberculosis. When you cough or sneeze, the transfer causes tuberculosis and the bacteria of tuberculosis spread through the air. If you are pregnant, you are also more likely to develop TB. Age is under 5 years or over 65 years old. Drink or inject drugs. Do not eat well. Once inside the body,TB can be inactive or active. Inactive TB is called latent TB. Active TB is called tuberculosis.

\section{Mathematical Model}

Firstly split host people represented with $T(t)$ in the form of four sections: susceptible persons $w(t)$,

$$
\begin{gathered}
\frac{d S(t)}{d t}=B N-\beta_{T} S I_{T}-\beta_{H} S I_{H}-\beta_{H T} S I_{H T}-(\xi+\mu) S-\varepsilon V-\eta R \\
\frac{d E_{T}}{d t}=\beta_{T} S I_{T}-\left(\mu+\chi_{T}+\alpha_{T}\right) E_{T} \\
\frac{d E_{H}}{d t}=\beta_{T} S I_{H}-\left(\mu+\chi_{H}+\alpha_{H}\right) E_{H} \\
\frac{d E_{H T}}{d t}=\beta_{H T} S I_{H T}-\left(\mu+\alpha_{H T}\right) E_{H T}+\chi_{T} E_{T}+\chi_{H} E_{H} \\
\frac{d I_{T}}{d t}=\alpha_{T} E_{T}-\left(\mu+d_{T}+\psi_{T}+\gamma_{T}\right) I_{T} \\
\frac{d I_{H}}{d t}=\alpha_{H} E_{H}-\left(\mu+\delta_{H}+\psi_{H}-\theta\right) I_{H}
\end{gathered}
$$




$$
\begin{aligned}
& \frac{d I_{H T}}{d t}=\alpha_{H T} E_{H T}-\left(\mu+\delta_{H T}+d_{T}-\psi_{T}-\psi_{H}-\theta\right) I_{H} \\
& \frac{d A_{H}}{d t}=\delta_{H} I_{H}-\left(\mu+d_{H}+\gamma_{H}\right) A_{H} \\
& \frac{d A_{H T}}{d t}=\delta_{H T} I_{H T}-\left(\mu+d_{H T}+\gamma_{H T}\right) A_{H T} \\
& \frac{d Q_{T}}{d t}=\gamma_{T} I_{T}-\left(\mu+d_{t}+\sigma_{T}\right) Q_{T} \\
& \frac{d Q_{H}}{d t}=\gamma_{H} A_{H}-\left(\mu+d_{H}+\sigma_{H}\right) Q_{H} \\
& \frac{d Q_{H T}}{d t}=\gamma_{H T} A_{H T}-\left(\mu+d_{H T}+\sigma_{H T}\right) Q_{H T} \\
& \frac{d R}{d t}=\sigma_{T} Q_{T}+\sigma_{H} Q_{H}+\sigma_{H T} Q_{H T}-(\mu+\eta) R \\
& \frac{d v}{d t}=\xi S-(\varepsilon+\mu) V
\end{aligned}
$$

and

$$
\begin{gathered}
N(t)=S(t)+E_{T}(t)+E_{H}(t)+E_{H T}(t)+I_{T}(t)+I_{H}(t)+I_{H T}(t)+A_{H}(t)+A_{H T}(t)+Q_{T}(t) \\
+Q_{H}(t)+Q_{H T}(t)+R(t)+V(t)
\end{gathered}
$$

Here

$$
\begin{gathered}
E_{T}(0)=2000 ; E_{H}(0)=2000 ; E_{H T}(0)=2000 ; I_{T}(0)=2000 ; I_{H}(0)=2000 ; I_{H T}(0)=2000 \\
A_{H}(0)=2000 ; A_{H T}(0)=2000 ; Q_{T}(0)=2000 ; Q_{H}(0)=2000 ; Q_{H T}(0)=2000 \\
R(0)=1000 ; V(0)=0 ; S(0)=93,000
\end{gathered}
$$




\section{Model PARAmeters AND its FORMUlation}

$:$

We divided the human population into six classes SEIQRV (Susceptible-Exposed-Infected-QuarantinedRecovered-Vaccinated) and the bird population into three classes $S_{b} E_{b} I_{b}$ (Susceptible-Exposed-Infected).The state variables and associated parameters of this model are given below. The state variables and associated parameters of co-infected model of HIV and TB

$\mathrm{S}(\mathrm{t})=$ Susceptible humans in time $\mathrm{t}, E_{T}(t)=$ Exposed humans with TB in time t, $E_{H}(t)=$ Exposed humans with HIV + in time t, $E_{H T}(t)=$ Exposed humans with TB and HIV + in time t, $I_{T}(t)=$ Infectious humans with TB in time t, $I_{H}(t)=$ Infectious humans with HIV + in time t, $I_{H T}(t)=$ Co-infectious humans with TB and $\mathrm{HIV}+$ in time t, $A_{H}(t)=$ AIDS infected humans in time t $A_{H T}(t)=$ AIDS and TB co-infected humans in time t, $Q_{T}(t)=$ Quarantined humans who are infected with TB in time t, $Q_{H}(t)=$ Quarantined humans who are infected with AIDS in time t, $Q_{H T}(t)=$ Quarantined humans who are co-infected with AIDS and TB in time $\mathrm{t}, R(t)=$ Recovered humans in time $\mathrm{t} V(t)=$ Vaccinated humans in time $\mathrm{t}, N(t)=$ Total human population in time t, $B=$ Birth rate of humans $\beta_{T}=$ Infectivity of TB, $\beta_{H}=$ Infectivity of HIV $+\beta_{H T}=$ Infectivity of both $\mathrm{TB}$ and $\mathrm{HIV}+, \eta=$ Rate of transmission from Recovered to Susceptible humans, $\gamma_{T}=$ Rate of transmission from TB infected humans to Quarantined humans with TB infected, $\gamma_{H}=$ Rate of transmission from AIDS infected humans to Quarantined humans with AIDS infected, $\gamma_{H T}=$ Rate of transmission from TB and AIDS Co-infected humans to Quarantined humans with TB and AIDS co- infected, $\alpha_{T}=$ Rate of transmission from Exposed with TB humans to Infected TB humans $\alpha_{H}=$ Rate of transmission from Exposed with HIV + humans to Infected HIV + humans, $\alpha_{H T}=$ Rate of transmission from Co-exposed with TB and HIV + humans to Co-infected with TB and HIV+ humans, $\sigma_{T}=$ Rate of transmission from Quarantined humans with TB infected to Recovered humans, $\sigma_{H}=$ Rate of transmission from Quarantined humans with AIDS infected to Recovered humans, $\sigma_{H T}=$ Rate of transmission from Quarantined humans with TB and AIDS co-infected to Recovered humans, $\xi=$ Rate of transmission from Vaccinated to Susceptible humans, $\varepsilon=$ Rate of transmission from Susceptible humans to Vaccinated humans, $\chi_{T}=$ Rate of transmission from TB infected humans to coinfected humans with TB and HIV,$+ \chi_{H}=$ Rate of transmission from HIV + infected humans to co-infected humans with TB and HIV,$+ \theta=$ Vertical transmission for HIV,$+ \mu=$ Natural death rate of humans, $\delta_{H}=$ Rate of transmission from HIV + infected humans to AIDS infected humans, $\delta_{H T}=$ Rate of transmission from TB and HIV + Co-infected humans to TB and AIDS Co-infected humans, $d_{t}=$ Death rate due to TB, $d_{H}=$ Death rate due to AIDS, $d_{H T}=$ Death rate due to TB and AIDS. Parameter values used in mathematical model: 


\begin{tabular}{|l|l|l|l|}
\hline Parameter & value & Parameter & value \\
\hline $\mathrm{N}$ & $1,00,000$ & $\chi_{H}$ & 0.3 \\
\hline $\mathrm{B}$ & 0.8 & $\delta_{H}$ & 0.6 \\
\hline$\beta_{T}$ & 0.8 & $\delta_{H T}$ & 0.7 \\
\hline$\beta_{H}$ & 0.85 & $\gamma_{T}$ & 0.64 \\
\hline$\beta_{H T}$ & 0.75 & $\gamma_{H}$ & 0.3 \\
\hline$\alpha_{T}$ & 0.65 & $\gamma_{H T}$ & 0.4 \\
\hline$\alpha_{H}$ & 0.7 & $d_{T}$ & 0.1 \\
\hline$\alpha_{H T}$ & 0.02 & $d_{H}$ & 0.5 \\
\hline$\chi_{T}$ & 0.2 & $d_{H T}$ & 0.6 \\
\hline$\sigma_{T}$ & 0.8 & $\psi_{T}$ & 0.08 \\
\hline$\sigma_{H}$ & 0.4 & $\psi_{H}$ & 0.2 \\
\hline$\sigma_{H T}$ & 0.1 & $\mu$ & 0.03 \\
\hline$\varepsilon$ & 0.05 & $\eta$ & 0.6 \\
\hline$\xi$ & 0.08 & $\theta$ & 0.001 \\
\hline
\end{tabular}

\section{Qualitative Analysis}

Disease Free point is

$$
\begin{gathered}
\left(S(t), E(t)_{T_{0}}, E(t)_{H_{0}}, E(t)_{H T_{0}}, I(t)_{T_{0}}, I(t)_{H_{0}}, I(t)_{H T_{0}}, A(t)_{H_{0}}, A(t)_{H T_{0}},\right. \\
\left.{ }_{0}, Q(t)_{T_{0}}, Q(t)_{H_{0}}, Q(t)_{H T_{0}}, R(t)_{0}, V(t)_{0}\right)=(1.438,0,0,0,0,0,0,0,0,0,0,0,0,5663.7)
\end{gathered}
$$

Hence, the endemic point is

$$
\begin{gathered}
\left(S_{E}, E(t)_{T_{E}}, E(t)_{H_{E}}, E(t)_{H T_{E}}, I(t)_{T_{E}}, I(t)_{H_{E}}, I(t)_{H T_{E}}, A(t)_{H_{E}}, A(t)_{H T_{E}}, Q(t)_{T_{E}}\right. \\
\left.Q(t)_{H_{E}}, Q(t)_{H T_{E}}, R(t)_{E}, V(t)_{E}\right)=\left(\frac{\left(\mu+\chi_{T}+\alpha_{T}\right)\left(\mu+d_{T}+\psi_{T}+\gamma_{T}\right)}{\beta_{T} \alpha_{T}}, 0,0,0,0,0,0,0,0,0,0,0,0\right. \\
\left.\frac{\xi B N}{(1+\epsilon \xi)}\right)
\end{gathered}
$$

\section{Preliminaries}

Definition 1: Suppose $f \in \mathrm{H}(\mathrm{c}, \mathrm{d}), \mathrm{d}>\mathrm{c}, \rho \in[1,1]$ : then the newly presented Caputo derivative of arbitrary [14] is given by

$$
\left(D_{t}^{\rho}\right) f(t)=\frac{L(\rho)}{1-\rho} \int_{c}^{t} f^{\prime}(t) \exp \left[-\rho \frac{t-x}{1-\rho}\right] d x
$$

where $L(\rho)$ is the normalization of the function satisfying the condition $L(0)=L(1)=1[17]$.

But, if $g \notin \mathrm{H}(\mathrm{c}, \mathrm{d})$ under this condition, the derivative is given follows: 
$\left(D_{t}^{\rho}\right) f(t)=\rho \frac{L(\rho)}{1-\rho} \int_{c}^{t}(f(t)-f(x)) \exp \left[-\rho \frac{t-x}{1-\rho}\right] d x$

Remark: If $\sigma=\frac{1-\rho}{\rho} \in[0,1]$, then the above equation assumes the form

$$
\left(D_{t}^{\rho}\right) f(t)=\frac{R(\sigma)}{\sigma} \int_{c}^{t}\left(f^{\prime}(t) \exp \left[-\frac{t-x}{\sigma}\right] d x, R(0)=R(\infty)=1\right.
$$

Morever

$$
\lim _{\sigma \rightarrow \infty} \frac{1}{\sigma} \exp \left[-\frac{t-x}{\sigma}\right]=\vartheta(x-t)
$$

Its is necessary to have the antiderivative associated with the new fractional derivative.

\section{Definition 02:}

Let us recall the well known definition of Caputo fractional derivative. Given $c>0, j \in H_{1}(0, c)$ and $0<\alpha<1$, the Caputo fractional derivative of $\mathrm{j}$ of order $\alpha$ is given by

$$
{ }^{C} D^{\alpha} j(t)=\frac{1}{\Gamma(1-\alpha)} \int_{0}^{t}(t-s)^{-\alpha}\left(j^{\prime}(s) d s, t>0\right.
$$

Fractional calculus and, in particular, Caputo fractional derivative, finds numerous applications in different areas of science.

By changing the kernel $(t-s)$ by the function $\exp (-\alpha(t-s) /(1-) \alpha)$ and $1 / \Gamma(1-\alpha) b y 1 / \sqrt{2 \pi\left(1-\alpha^{2}\right)}$ one obtains the new Caputo-Fabrizio fractional derivative of order $0<\alpha<1$. That is

$$
{ }^{C F} D^{\alpha} j(t)=\frac{2-\alpha) M(\alpha)}{2(1-\alpha)} \int_{0}^{t}\left(\exp \left(-\frac{\alpha}{1-\alpha}(t-s)\right) j^{\prime}(s) d s\right.
$$

where $M(\alpha)$ is a normalization constant depending on $\alpha$. According to the new definition, it is clear that if $\mathrm{j}$ is a constant function, then ${ }^{C} D^{\alpha} j(t)=0$ as in the usual Caputo derivative. The main difference between old and new definition is that, contrary to the old definition, the new kernel has no singularity for $t=s$. It is well known that Laplace Transform plays an important role in the study of ordinary differential equations. In the case of this new fractional definition, it is also known that, for $0<\alpha<1$

$$
\mathcal{L}\left\{{ }^{C F} D^{\alpha} j(t)\right\} s=\frac{(2-\alpha) M(\alpha)}{2(s+\alpha(1-s))}(s \mathcal{L}\{j(t)\}(s)-j(0))
$$

where $\mathcal{L}\{j\}$ denotes the Laplace Transform of function $j$. So, it is clear that if we work with CaputoFabrizio derivative, Laplace Transform will also be a very useful tool.

Theorem 1: The closed set D is bounded and positive invariant.

Proof: $\frac{d N}{d t} \leq B-\mu N$

So Nis bounded above by $\frac{B}{\mu}$.

Hence $\frac{d N}{d t}<0$ whenver $(t)>\frac{B}{\mu}$

On simplification, we have 
$N(t) \leq N(0) e^{\mu t}+\frac{B}{\mu}\left(1-e^{\mu t}\right)$ As $t \rightarrow \infty, e^{\mu t} \rightarrow 0$ and so $\lim _{t \rightarrow \infty} N(t) \leq \frac{B}{\mu}$ Thus, D is bounded and positively invariant in $R_{+}^{14}$

\section{Caputo fractional order model}

Converting the epidemic model $(2.1-2.14)$ subject to the initial condition $(2.15)$ into fractional order model by using caputo definition, then Applying the Laplace transform on equation we get

$$
\begin{aligned}
& \mathcal{L}\left\{{ }_{0}^{C} D_{t}^{\alpha} S(t)\right\}=B N \mathcal{L}\{1\}-\beta_{T} \mathcal{L}\left\{S I_{T}\right\}-\beta_{H} \mathcal{L}\left\{S I_{H}\right\}-\beta_{H T} \mathcal{L}\left\{S I_{H T}\right\} \\
& -(\xi+\mu) \mathcal{L}\{S\}-\varepsilon \mathcal{L}\{V\}-\eta \mathcal{L}\{R\} \\
& \mathcal{L}\left\{{ }_{0}^{C} D_{t}^{\alpha} E(t)_{T}\right\}=\beta_{T} \mathcal{L}\left\{S I_{T}\right\}-\left(\mu+\chi_{T}+\alpha_{T}\right) \mathcal{L}\left\{E_{T}\right\} \\
& \mathcal{L}\left\{{ }_{0}^{C} D_{t}^{\alpha} E(t)_{H}\right\}=\beta_{T} \mathcal{L}\left\{S I_{H}\right\}-\left(\mu+\chi_{H}+\alpha_{H}\right) \mathcal{L}\left\{E_{H}\right\} \\
& \mathcal{L}\left\{{ }_{0}^{C} D_{t}^{\alpha} E(t)_{H T}\right\}=\beta_{H T} \mathcal{L}\left\{S I_{H T}\right\}-\left(\mu+\alpha_{H T}\right) \mathcal{L}\left\{E_{H T}\right\}+\chi_{T} \mathcal{L}\left\{E_{T}\right\} \\
& +\chi_{H} \mathcal{L}\left\{E_{H}\right\} \\
& \mathcal{L}\left\{{ }_{0}^{C} D_{t}^{\alpha} I(t)_{T}\right\}=\alpha_{T} \mathcal{L}\left\{E_{T}\right\}-\left(\mu+d_{T}+\psi_{T}+\gamma_{T}\right) \mathcal{L}\left\{I_{T}\right\} \\
& \mathcal{L}\left\{{ }_{0}^{C} D_{t}^{\alpha} I(t)_{H}\right\}=\alpha_{H} \mathcal{L}\left\{E_{H}\right\}-\left(\mu+\delta_{H}+\psi_{H}-\theta\right) \mathcal{L}\left\{I_{H}\right\} \\
& \mathcal{L}\left\{{ }_{0}^{C} D_{t}^{\alpha} I(t)_{H T}\right\}=\alpha_{H T} \mathcal{L}\left\{E_{H T}\right\}-\left(\mu+\delta_{H T}+d_{T}-\psi_{T}-\psi_{H}-\theta\right) \mathcal{L}\left\{I_{H T}\right\} \\
& \mathcal{L}\left\{{ }_{0}^{C} D_{t}^{\alpha} A(t)_{H}\right\}=\delta_{H} \mathcal{L}\left\{I_{H}\right\}-\left(\mu+d_{H}+\gamma_{H}\right) \mathcal{L}\left\{A_{H}\right\} \\
& \mathcal{L}\left\{{ }_{0}^{C} D_{t}^{\alpha} A(t)_{H T}\right\}=\delta_{H T} \mathcal{L}\left\{I_{H T}\right\}-\left(\mu+d_{H T}+\gamma_{H T}\right) \mathcal{L}\left\{A_{H T}\right\} \\
& \mathcal{L}\left\{{ }_{0}^{C} D_{t}^{\alpha} Q(t)_{T}\right\}=\gamma_{T} \mathcal{L}\left\{I_{T}\right\}-\left(\mu+d_{t}+\sigma_{T}\right) \mathcal{L}\left\{Q_{T}\right\} \\
& \mathcal{L}\left\{{ }_{0}^{C} D_{t}^{\alpha} Q(t)_{H}\right\}=\gamma_{H} \mathcal{L}\left\{A_{H}\right\}-\left(\mu+d_{H}+\sigma_{H}\right) \mathcal{L}\left\{Q_{H}\right\} \\
& \mathcal{L}\left\{{ }_{0}^{C} D_{t}^{\alpha} Q(t)_{H T}\right\}=\gamma_{H T} \mathcal{L}\left\{A_{H T}\right\}-\left(\mu+d_{H T}+\sigma_{H T}\right) \mathcal{L}\left\{Q_{H T}\right\} \\
& \mathcal{L}\left\{{ }_{0}^{C} D_{t}^{\alpha} R(t)\right\}=\sigma_{T} \mathcal{L}\left\{Q_{T}\right\}+\sigma_{H} \mathcal{L}\left\{Q_{H}\right\}+\sigma_{H T} \mathcal{L}\left\{Q_{H T}\right\}-(\mu+\eta) \mathcal{L}\{R\} \\
& \mathcal{L}\left\{{ }_{0}^{C} D_{t}^{\alpha} V(t)\right\}=\xi \mathcal{L}\{S\}-(\varepsilon+\mu) \mathcal{L}\{V\}
\end{aligned}
$$


The series solution is as follows

$$
\begin{aligned}
& S(t)=93,000-446,33,830 \frac{t^{\alpha}}{\alpha !}+2.1436 \times 10^{12} \frac{t^{2 \alpha}}{2 \alpha !}+106,62400 \frac{t^{3 \alpha}}{3 \alpha !} \frac{2}{\alpha !}+6.016 \times 10^{15} \frac{t^{3 \alpha}}{3 \alpha !}+\ldots \ldots \\
& E_{T}(t)=2000+148,798,240 \frac{t^{\alpha}}{\alpha !}-714472000000 \frac{t^{2 \alpha}}{2 \alpha !}-6.145 \times 10^{11} \frac{t^{3 \alpha}}{3 \alpha !}-25,600,000 \frac{t^{3 \alpha}}{3 \alpha !} \frac{2}{\alpha !} \\
& E_{H}(t)=2000+158097940 \frac{t^{\alpha}}{\alpha !}-7.59164 \times 10^{11} \frac{t^{2 \alpha}}{2 \alpha !}-6.529 \times 10^{11} \frac{t^{3 \alpha}}{3 \alpha !}-\frac{17544,000}{3 \alpha !} \frac{t^{3 \alpha} 2}{\alpha !}+\ldots \ldots \\
& E_{H T}(t)=2000+139,500,900 \frac{t^{\alpha}}{\alpha !}-6.6958 \times 10^{11} \frac{t^{2 \alpha}}{2 \alpha !}-5.759 \times 10^{11} \frac{t^{3 \alpha}}{3 \alpha !}-63,480,000 \frac{t^{3 \alpha}}{3 \alpha !} \frac{2}{\alpha !}+\ldots \ldots \\
& I_{T}(t)=2000-400 \frac{t^{\alpha}}{\alpha !}+96,719,192 \frac{t^{2 \alpha}}{2 \alpha !}+83,200,000 \frac{t^{3 \alpha}}{3 \alpha !}+\ldots \ldots \ldots \\
& I_{H}(t)=2000-258 \frac{t^{\alpha}}{\alpha !}+110,668,794.3 \frac{t^{2 \alpha}}{2 \alpha !}+\frac{95,200,000}{3 \alpha !} t^{3 \alpha}+\ldots . \\
& I_{H T}(t)=2000-1058 \frac{t^{\alpha}}{\alpha !}+2,790,598.842 \frac{t^{2 \alpha}}{2 \alpha !}+\frac{2,400,000}{3 \alpha !} t^{3 \alpha}+\ldots \ldots \ldots \\
& A_{H}(t)=2000-460 \frac{t^{\alpha}}{\alpha !}+227 \frac{t^{2 \alpha}}{2 \alpha !}+\ldots \ldots \\
& A_{H T}(t)=2000-660 \frac{t^{\alpha}}{\alpha !}-60.8 \frac{t^{2 \alpha}}{2 \alpha !}+\ldots \\
& Q_{T}(t)=2000-580 \frac{t^{\alpha}}{\alpha !}+283.4 \frac{t^{2 \alpha}}{\alpha !}+\ldots \ldots \\
& Q_{H}(A T)=2000-1260 \frac{t^{\alpha}}{\alpha !}+1033.8 \frac{t^{2 \alpha}}{2 \alpha !}+\ldots . \\
& Q_{H T}(t)=2000-660 \frac{t^{\alpha}}{\alpha !}+217.8 \frac{t^{2 \alpha}}{2 \alpha !}+\ldots \ldots \\
& R(t)=1000+1970 \frac{t^{\alpha}}{\alpha !}-2275.1 \frac{t^{2 \alpha}}{2 \alpha !}+\ldots \ldots \\
& V(t)=7440 \frac{t^{\alpha}}{\alpha !}-37,707,061.6 \frac{t^{2 \alpha}}{2 \alpha !}-30,721,216 \frac{t^{3 \alpha}}{3 \alpha !}+\ldots \ldots .
\end{aligned}
$$

\section{Fractional Order Model with Caputo Febrizio Sense}

The fractional order model of the system (2.1-2.14) by using caputo Febrizio derivative is given as.

$$
\begin{gathered}
{ }_{0}^{C F} D_{t}^{\alpha} S(t)=B N-\beta_{T} S I_{T}-\beta_{H} S I_{H}-\beta_{H T} S I_{H T}-(\xi+\mu) S-\varepsilon V-\eta R \\
{ }_{0}^{C F} D_{t}^{\alpha} E(t)_{T}=\beta_{T} S I_{T}-\left(\mu+\chi_{T}+\alpha_{T}\right) E_{T} \\
{ }_{0}^{C F} D_{t}^{\alpha} E(t)_{H}=\beta_{T} S I_{H}-\left(\mu+\chi_{H}+\alpha_{H}\right) E_{H} \\
{ }_{0}^{C F} D_{t}^{\alpha} E(t)_{H T}=\beta_{H T} S I_{H T}-\left(\mu+\alpha_{H T}\right) E_{H T}+\chi_{T} E_{T}+\chi_{H} E_{H} \\
{ }_{0}^{C F} D_{t}^{\alpha} I(t)_{T}=\alpha_{T} E_{T}-\left(\mu+d_{T}+\psi_{T}+\gamma_{T}\right) I_{T} \\
{ }_{0}^{C F} D_{t}^{\alpha} I(t)_{H}=\alpha_{H} E_{H}-\left(\mu+\delta_{H}+\psi_{H}-\theta\right) I_{H}
\end{gathered}
$$




$$
\begin{gathered}
{ }_{0}^{C F} D_{t}^{\alpha} I(t)_{H T}=\alpha_{H T} E_{H T}-\left(\mu+\delta_{H T}+d_{T}-\psi_{T}-\psi_{H}-\theta\right) I_{H T} \\
{ }_{0}^{C F} D_{t}^{\alpha} A(t)_{H}=\delta_{H} I_{H}-\left(\mu+d_{H}+\gamma_{H}\right) A_{H} \\
{ }_{0}^{C F} D_{t}^{\alpha} A(t)_{H T}=\delta_{H T} I_{H T}-\left(\mu+d_{H T}+\gamma_{H T}\right) A_{H T} \\
{ }_{0}^{C F} D_{t}^{\alpha} Q(t)_{T}=\gamma_{T} I_{T}-\left(\mu+d_{t}+\sigma_{T}\right) Q_{T} \\
{ }_{0}^{C F} D_{t}^{\alpha} Q(t)_{H}=\gamma_{H} A_{H}-\left(\mu+d_{H}+\sigma_{H}\right) Q_{H} \\
{ }_{0}^{C F} D_{t}^{\alpha} Q(t)_{H T}=\gamma_{H T} A_{H T}-\left(\mu+d_{H T}+\sigma_{H T}\right) Q_{H T} \\
{ }_{0}^{C F} D_{t}^{\alpha} R(t)=\sigma_{T} Q_{T}+\sigma_{H} Q_{H}+\sigma_{H T} Q_{H T}-(\mu+\eta) R \\
{ }_{0}^{C F} D_{t}^{\alpha} V(t)=\xi S-(\varepsilon+\mu) V
\end{gathered}
$$

The series solution is as follows

$$
\begin{array}{rl}
S(t)=9300- & 446330830(1-\alpha)+2.14239 \times 10^{12}(1-\alpha)^{2}+1.84324 \times 10^{12}(1-\alpha)^{3}-1182(1-\alpha) \\
+ & 446490830 \alpha+4.31477 \times 10^{12} \alpha(1-\alpha)-1182 \alpha^{2}+5.52973 \times 10^{12} \alpha(1-\alpha)^{2} \\
+ & \left.2.30403435 \times 10^{12} \alpha^{2}(1-\alpha)\right\} t+2.14258 \times 10^{12} \alpha^{2} t^{2}+1.53607 \times 10^{11} \alpha^{3} t^{3} \\
E_{T}(t)= & 2000+148798240(1-\alpha)-7.14242 \times 10^{11}(1-\alpha)^{2}-6.145 \times 10^{11}(1-\alpha)^{3} \\
+ & \left\{-1.4284 \times 10^{12} \alpha(1-\alpha)+148798240 \alpha-1.8435 \times 10^{12} \alpha(1-\alpha)^{2}\right\} t \\
\{- & \left.7.14306 \times 10^{11} \alpha^{2}-7.6815 \times 10^{11} \alpha^{2}(1-\alpha)\right\} t^{2}-5.1218 \times 10^{10} \alpha^{3} t^{3} \\
E_{H}(t)= & 2000+1158100000(1-\alpha)-7.5892 \times 10^{11}(1-\alpha)^{2}-6.5294 \times 10^{11}(1-\alpha)^{3} \\
+ & \left\{-1.51783 \times 10^{12} \alpha(1-\alpha)+158100000 \alpha-1.958 \times 10^{12} \alpha(1-\alpha)^{2}\right\} t \\
& \left\{-7.5899 \times 10^{11} \alpha^{2}-8.16 \times 10^{11} \alpha^{2}(1-\alpha)\right\} t^{2}-5.442 \times 10^{10} \alpha^{3} t^{3} \\
E_{H T}(t)= & 2000+139500000(1-\alpha)-6.6888 \times 10^{11}(1-\alpha)^{2}-5.75 \times 10^{11}(1-\alpha)^{3} \\
+ & \left\{-1.33896 \times 10^{12} \alpha(1-\alpha)+139500000 \alpha-1.727 \times 10^{12} \alpha(1-\alpha)^{2}\right\} t \\
& \left\{-3.3444 \times 10^{11} \alpha^{2}-7.1875 \times 10^{11} \alpha^{2}(1-\alpha)\right\} t^{2}-4.792 \times 10^{10} \alpha^{3} t^{3} \\
I_{H}(t)==2000-258(1-\alpha)+110670213.9(1-\alpha)^{2}+95200000(1-\alpha)^{3}+\{221340427.8 \alpha(1-\alpha)-258 \alpha & \\
+600000 & \left.\alpha(1-\alpha)^{2}\right\} t+\left\{55335106.95 \alpha^{2}+102935107 \alpha^{2}(1-\alpha)\right\} t^{2}+15866666.67 \alpha^{3} t^{3} \\
\left.+249600000 \alpha(1-\alpha)^{2}\right\} t+\left\{48359598 \alpha^{2}+124800000 \alpha^{2}(1-\alpha)\right\} t^{2}+13866666.67 \alpha^{3} t^{3} & \\
I_{T}(t)==2000 & 400(1-\alpha)+96719196(1-\alpha)^{2}+83200000(1-\alpha)^{3}+\{193438392 \alpha(1-\alpha)-400 \alpha
\end{array}
$$




$$
\begin{gathered}
I_{H T}(t)==2000-1058(1-\alpha)+2790580.842(1-\alpha)^{2}+2400000(1-\alpha)^{3}+\{5581161.684 \alpha(1-\alpha) \\
\left.-1058 \alpha+7200000 \alpha(1-\alpha)^{2}\right\} t+\left\{1395290421 \alpha^{2}+3600000 \alpha^{2}(1-\alpha)\right\} t^{2}+400000 \alpha^{3} t^{3} \\
\left.A_{(} H\right)(t)=2000-460(1-\alpha)+227(1-\alpha)^{2}+\{456 \alpha(1-\alpha)-460 \alpha\} t+113.5 \alpha^{2} t^{2} \\
\left.A_{(} H T\right)(t)=2000-660(1-\alpha)-60.8(1-\alpha)^{2}-\{121.6 \alpha(1-\alpha)+660 \alpha\} t-30.4 \alpha^{2} t^{2} \\
\left.Q_{(} T\right)(t)=2000-580(1-\alpha)+283.4(1-\alpha)^{2}+\{566.8 \alpha(1-\alpha)-580 \alpha\} t+141.7 \alpha^{2} t^{2} \\
\left.Q_{(} H\right)(t)=2000-1260(1-\alpha)+1033.8(1-\alpha)^{2}+\{2067.6 \alpha(1-\alpha)-1260 \alpha\} t+516.9 \alpha^{2} t^{2} \\
\left.Q_{(} H T\right)(t)=2000-660(1-\alpha)+217.8(1-\alpha)^{2}+\{435.6 \alpha(1-\alpha)-660 \alpha\} t+108.9 \alpha^{2} t^{2} \\
R(t)=1000+1970(1-\alpha)-2275.5(1-\alpha)^{2}+\{-4550.5 \alpha(1-\alpha)+1970 \alpha\} t-1137.5 \alpha^{2} t^{2} \\
V(t))==7440(1-\alpha)-35707061.6(1-\alpha)^{2}-3072192(1-\alpha)^{3}+\{-7129893.2 \alpha(1-\alpha)+7440 \alpha \\
\end{gathered}
$$

\section{Numerical Results and Discussion}

The mathematical analysis of SEICR epidemic model with nonlinear system of differential equation has been presented. Firstly we determined the disease free equilibrium point of fractional order model.For the reliable investigation, evaluation is made for different values of $\alpha$.Hence from figure 1 when we increase the parameter value the rate of Susceptible humans will be decrease.From Figs.2-4 we observed solution gives better converges to steady state for Exposed by increasing the fractional values of $\alpha$, while from Figs.5-9 the compartments converges fastly by putting different values of $\alpha$.Finally in the last fig. the Vaccinated humans in time $\mathrm{t}$ be increased by decreased the values of $\alpha$. The Laplace Adomian decomposition method is an approximate solution in term of infinite power series.Hence we can easily observed the steady state solution at fr5actinal order derivative is better, reliable and more effective reasults as compared to ordinary at $\alpha=1$. 


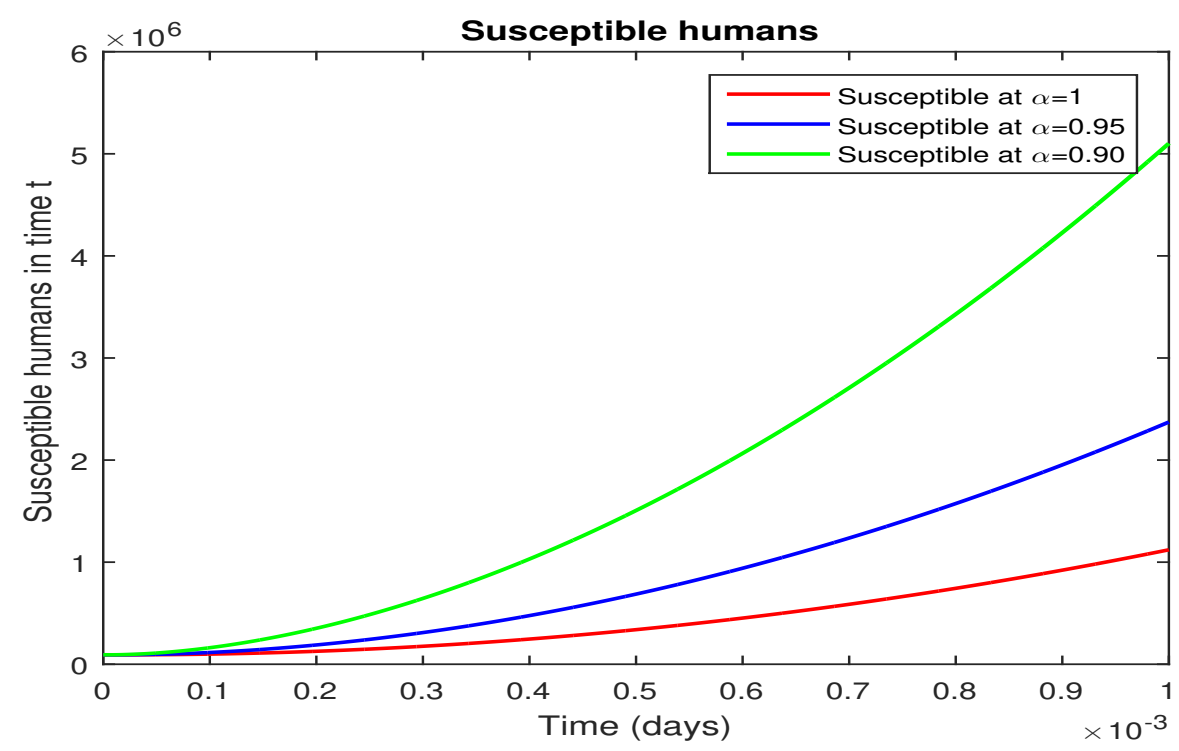

Figure 1. Numerical Solutions for Susceptible humans $S(t)$ in time $t$ at different values of $\alpha$.

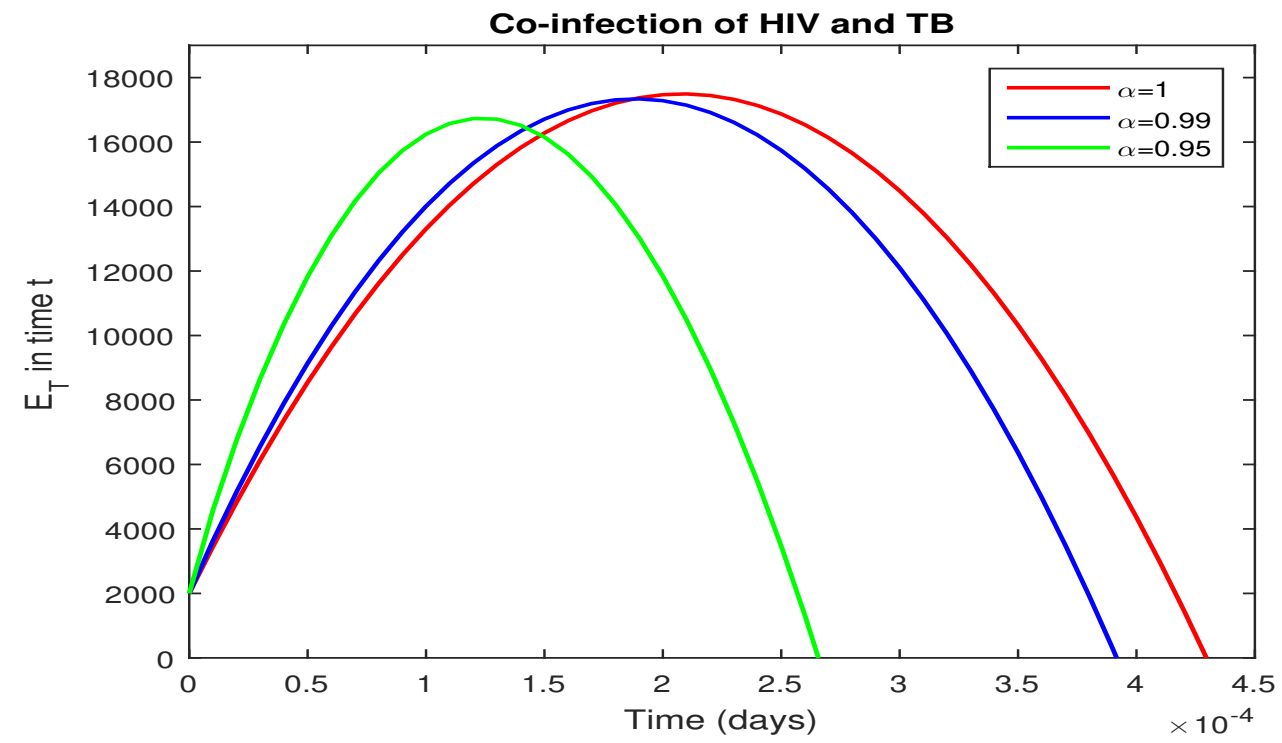

Figure 2. Numerical Solutions for Exposed humans with TB $E_{T}(t)$ in time $t$ at different values of $\alpha$. 


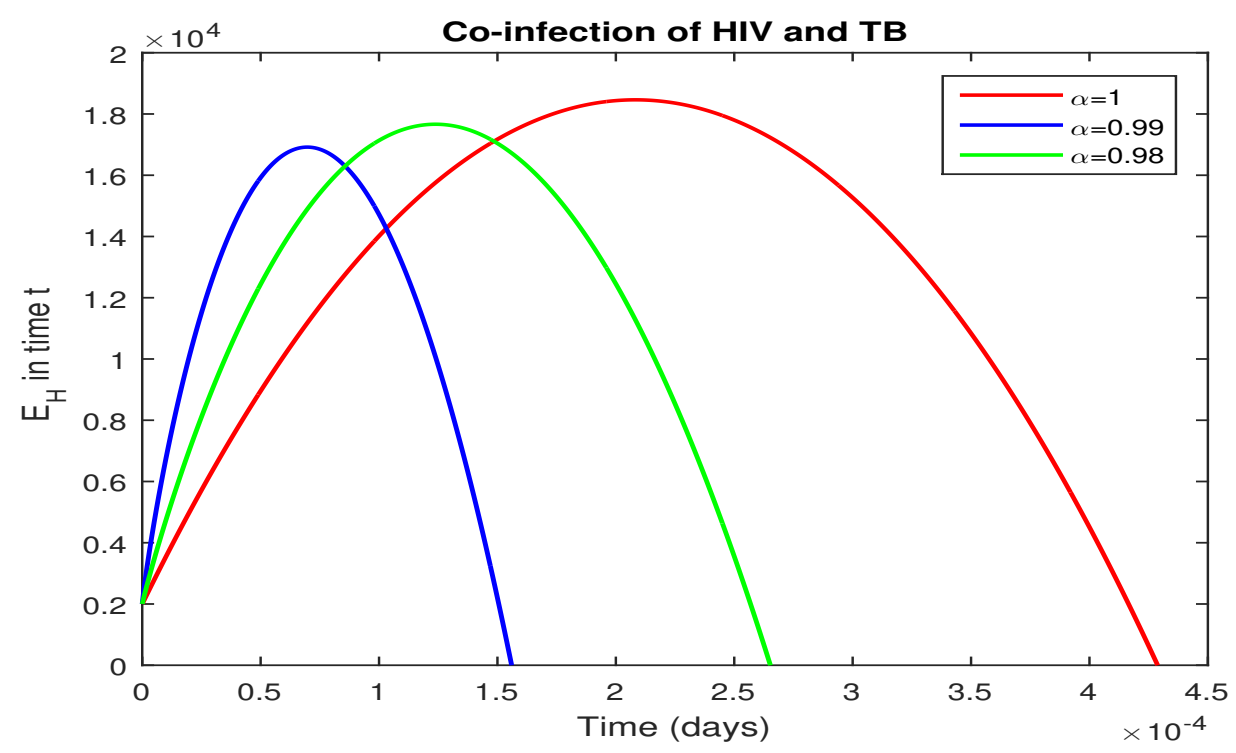

Figure 3. Numerical Solutions for Exposed humans with AIDS $E_{H}(t)$ in time $t$ at different values of $\alpha$.

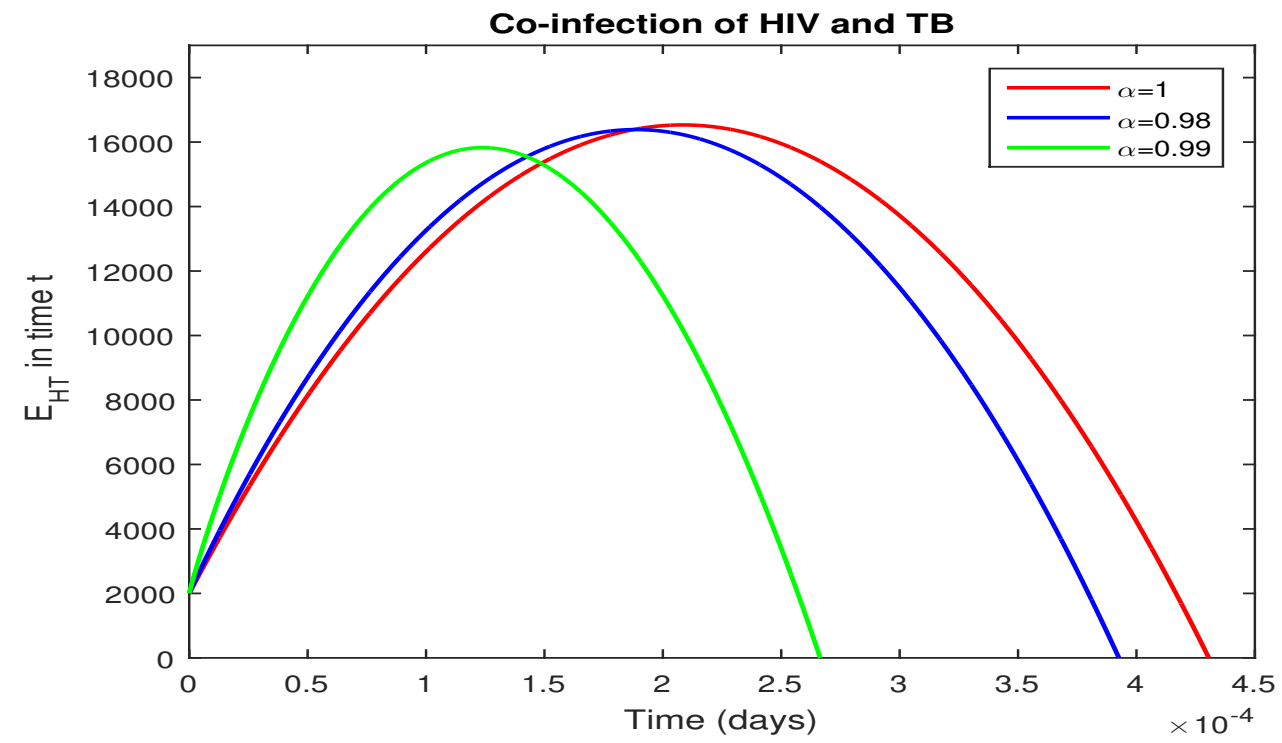

Figure 4. Numerical Solutions for Exposed humans with TB and HIV $E_{H T}(t)$ in time $t$ at different values of $\alpha$. 


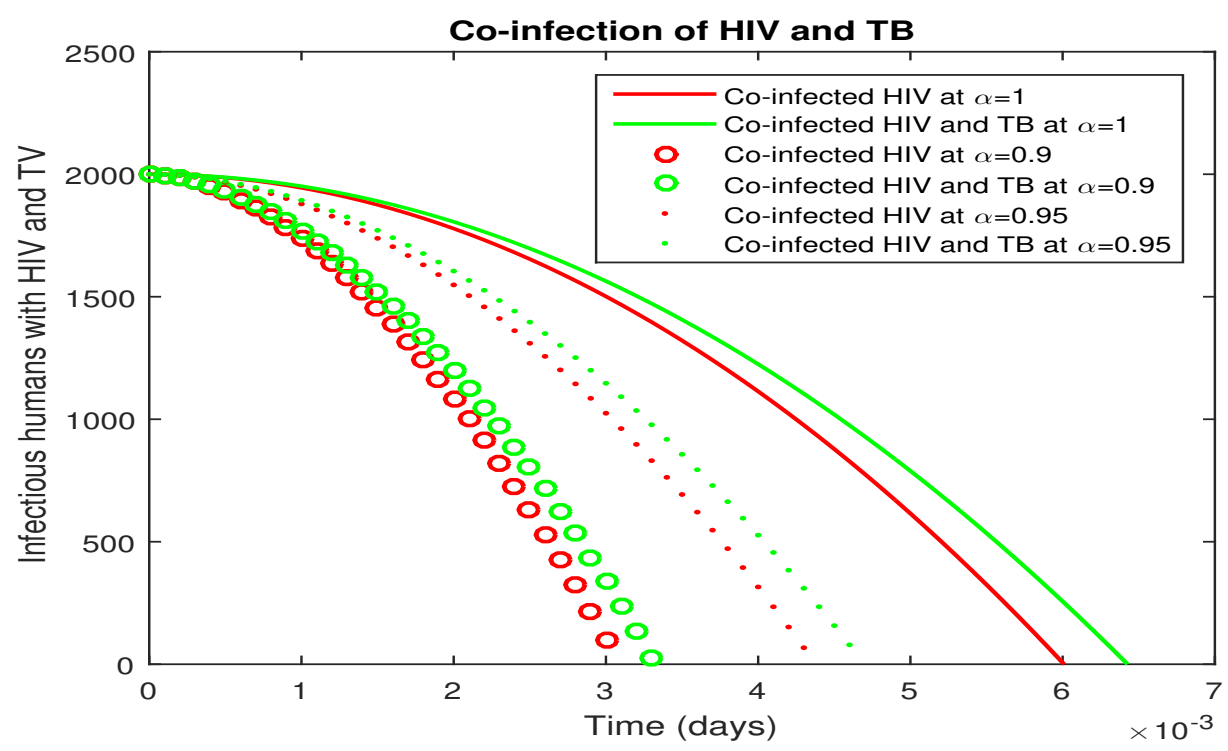

FiguRE 5. Numerical Solutions for Infectious humans with TB and HIV $I_{H}(t)$ in time $t$ at different values of $\alpha$.

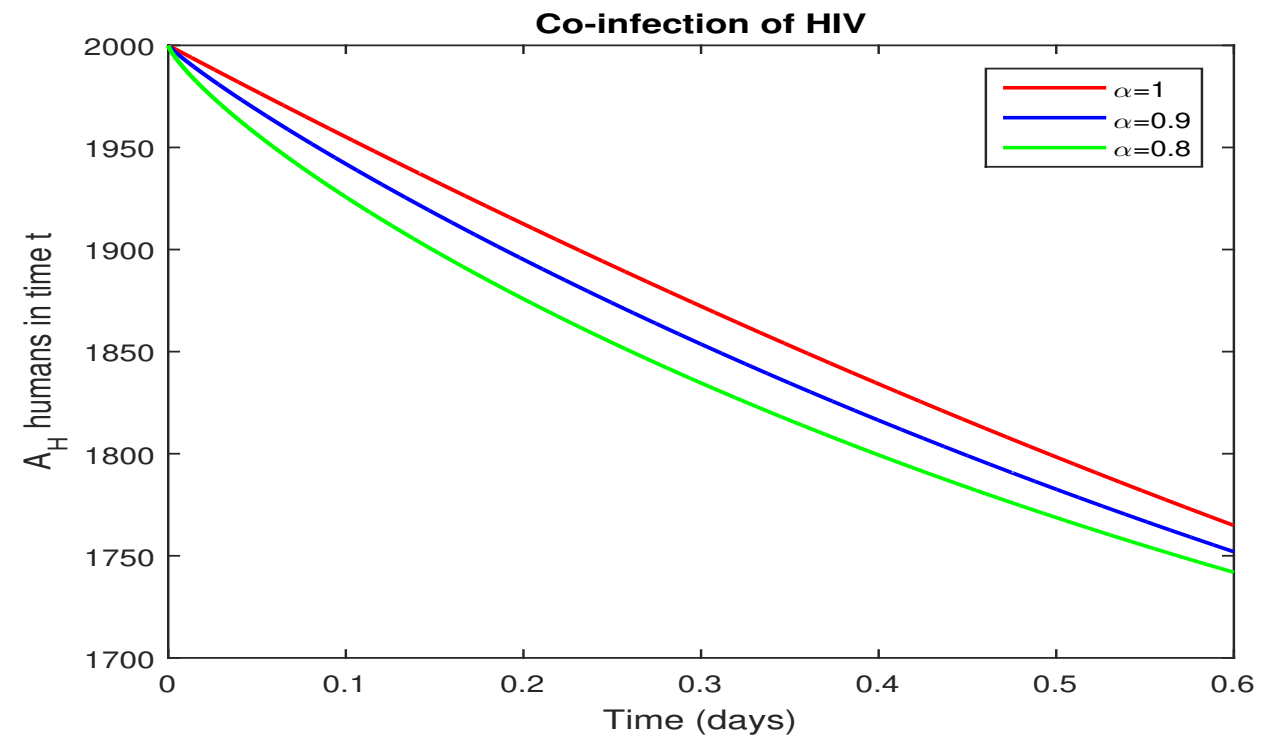

Figure 6. Numerical Solutions for AIDS infected humans $A_{H}(t)$ in time $t$ (year) at different values of $\alpha$. 


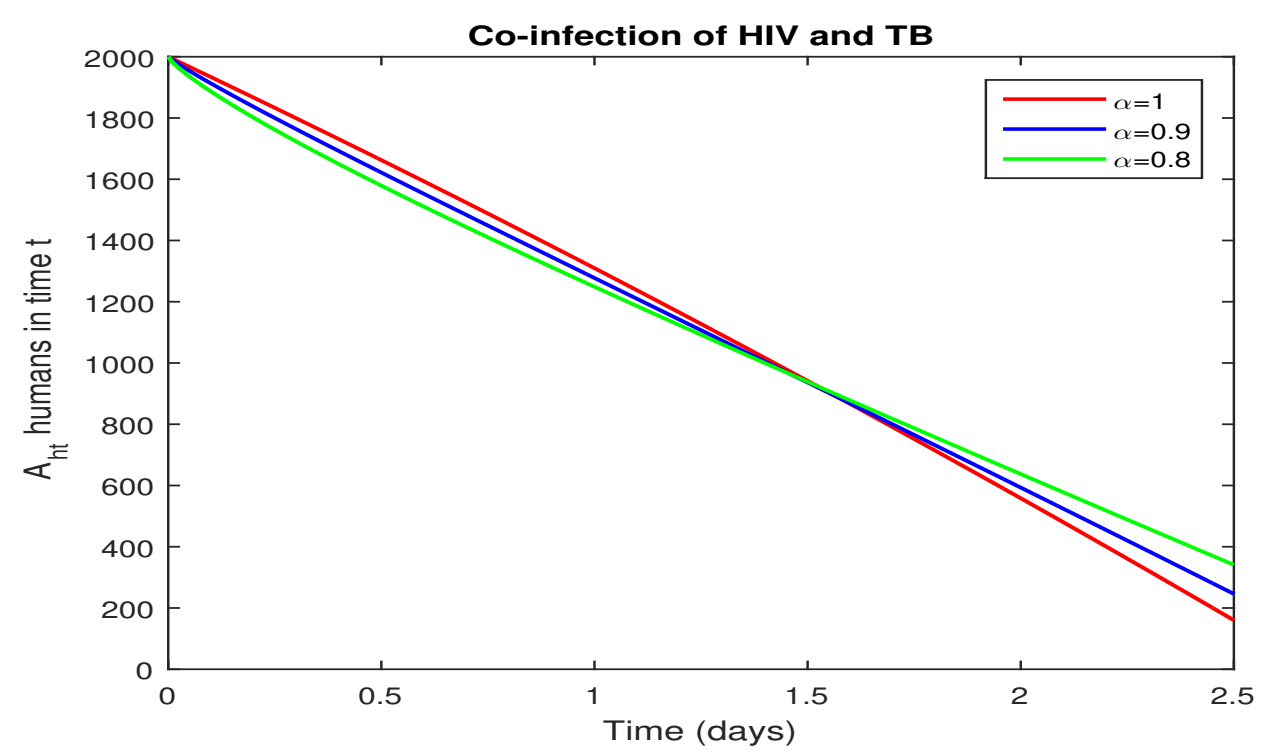

Figure 7. Numerical Solutions for AIDS and TB co-infected humans $A_{H T}(t)$ in time $t$ (year) at different values of $\alpha$.

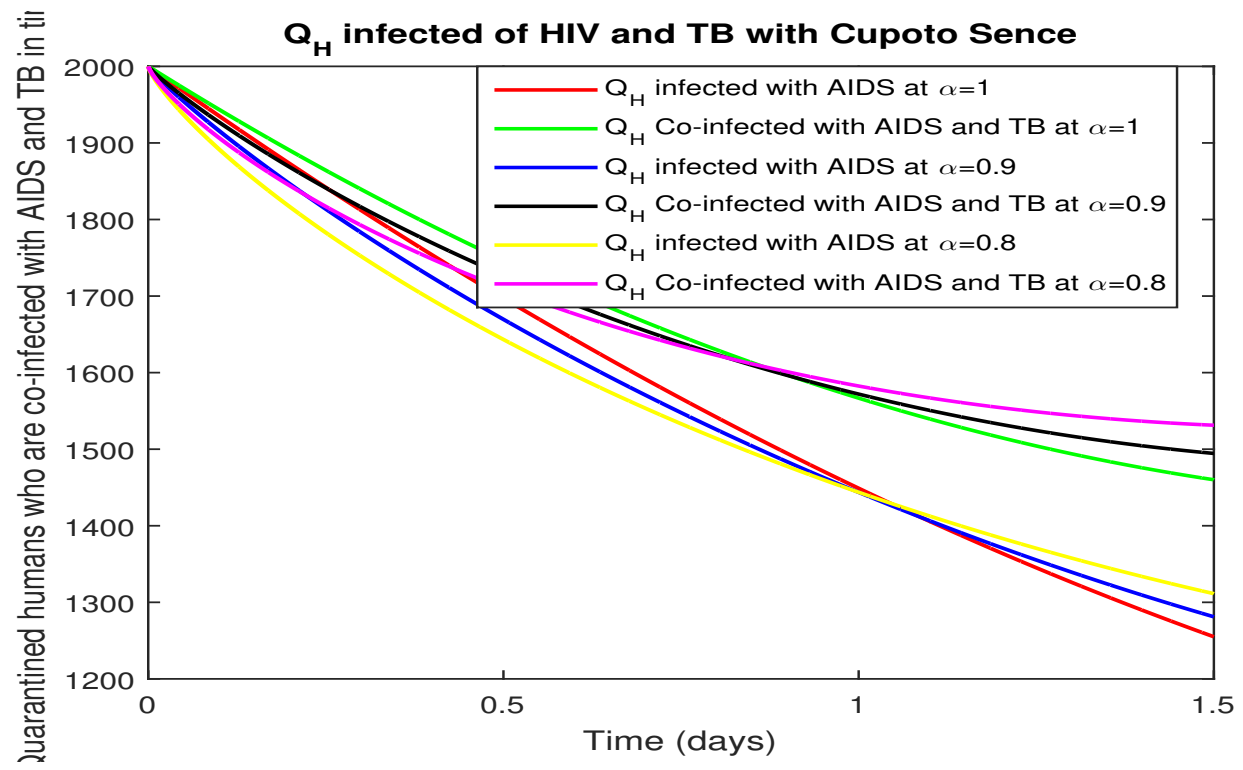

Figure 8. Numerical Solutions for Quarantined humans who are co-infected with AIDS and TB $Q_{H T}(t)$ in time $t$ (year) at different values of $\alpha$. 


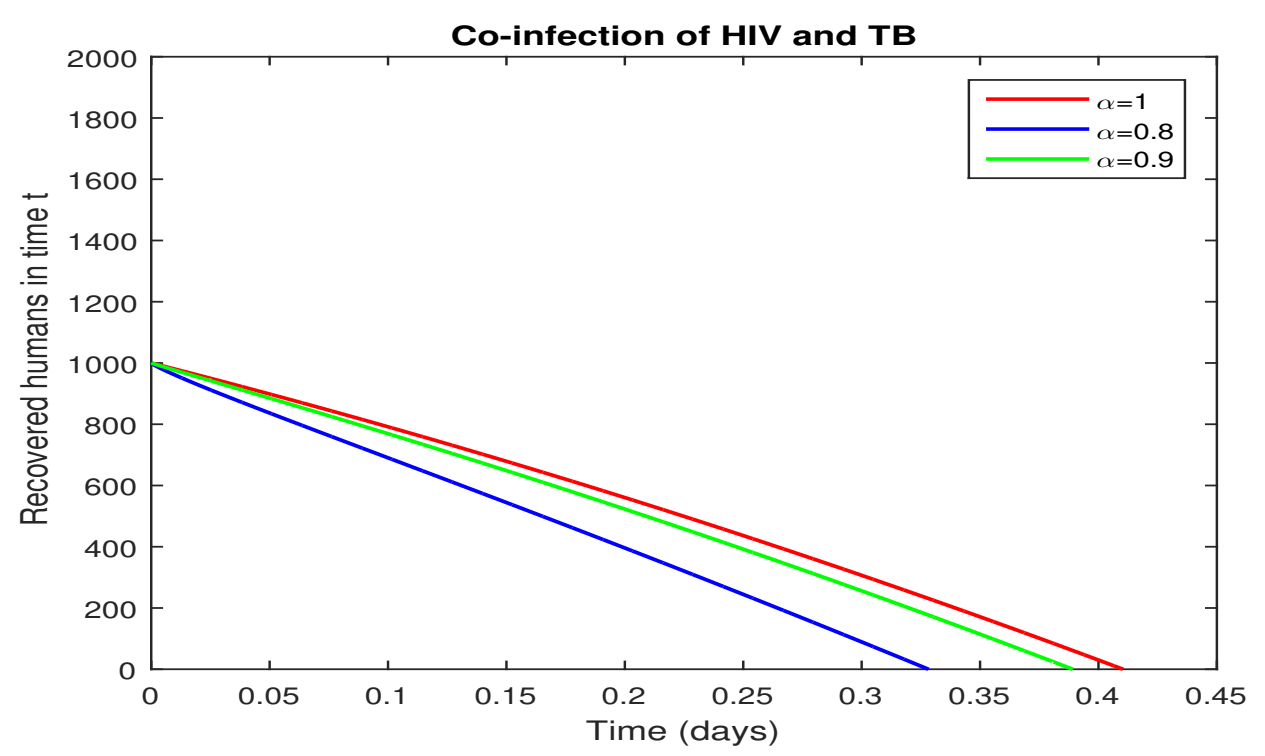

Figure 9. Numerical Solutions for Recovered humans $R(t)$ in time $t$ at different values of $\alpha$.

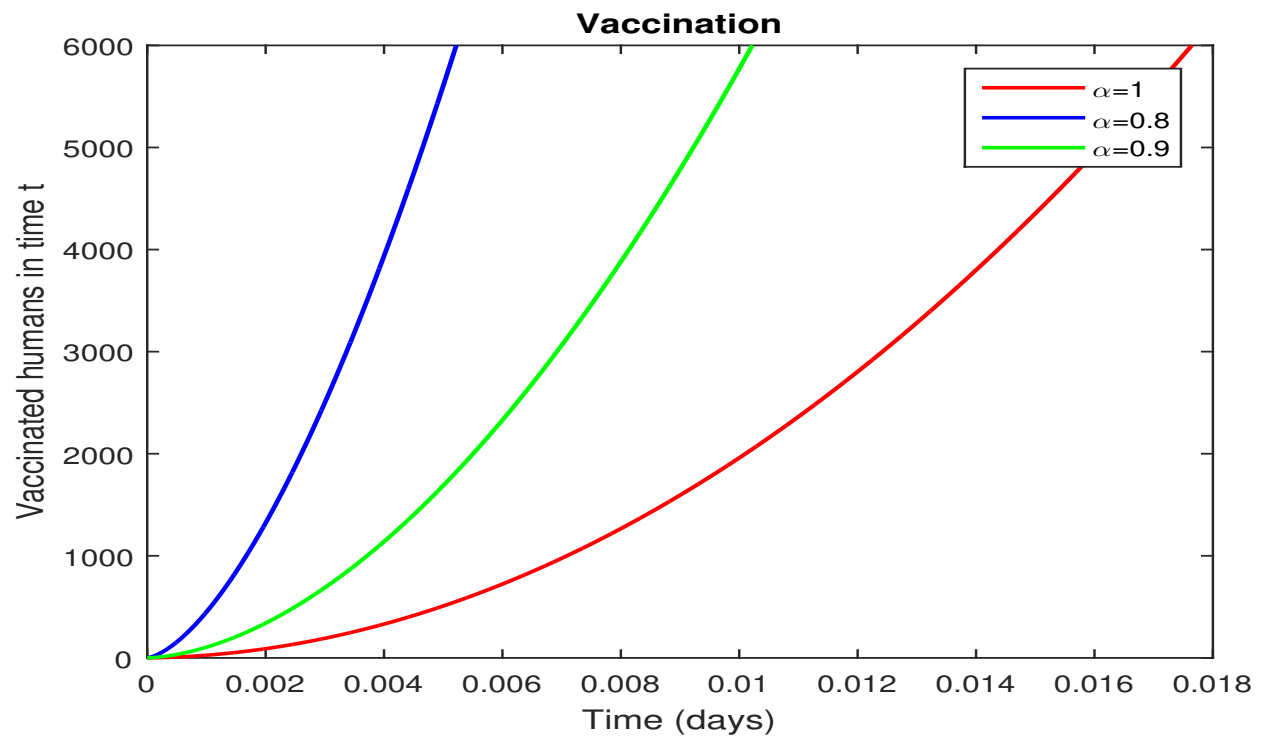

Figure 10. Numerical Solutions for Vaccinated humans $V(t)$ in time $t$ at different values of $\alpha$.

\section{Conclusion}

We developed a scheme for the solution of Co-Infection of TB and HIV by using Laplace Adomian decomposition method. The well known Co-Infection of TB and HIV model namely Susceptible population, Exposed population, Co-infectious population, Quarantined population, Recovered population and Vaccinated is considered with and without demographic effects. We can solved the detail model of Co-Infection of TB and HIV with fractional order derivative with Caputo sense and Caputo fabrizio sense and we use the 
Laplace Adomian Decomposition method to solve the model. It is also observed that to eliminate disease, it is not necessary to vaccinate whole of population. The effect of fractional parameter on our obtained solutions is presented through Graphs It is worthy to observe that fractional order derivative show significant changes and memory effect as compared to ordinary derivative.

Conflicts of Interest: The author(s) declare that there are no conflicts of interest regarding the publication of this paper.

\section{REFERENCES}

[1] N. T. J. Bailey, The mathematical approach to biology and medicine, John Wiley, New York, 1967.

[2] E. J. Williams, The development of biomathematical models. 36th session, International Statistical Institute, Sydney, 1967.

[3] H. F. von Helmholtz, On the sensations of tone as a physiological basis for the theory of music. (2nd ed.) Dover Publics, New York, 1954.

[4] S. Freud, Project on a Scientific Psychology, in The Standard Edition of the Complete Psychological Works of Sigmund Freud, Vol I, pp 283-346. Hogarth Press, London, 1966.

[5] N. Wiener, Cybernetics, (2nd ed.) MIT Press, Cambridge, 1961.

[6] R. Thom, Structural Stability and Morphogenesis, Westview Press; New Ed edition, 2001.

[7] G. Edelmann, Neural Darwinism: The Theory of Neuronal Group Selection, Basic Books, New York, 1987.

[8] R. Penrose, The Emperors New Mind: Concerning Computers, Minds, and the Laws of Physics, Amer. J. Phys. 58(1990), 1214.

[9] https://www.unaids.org/en.

[10] S. Suchindran, Is HIV infection a Risk Factor for Multi-Drug Resistant Tuberculosis? A Systematic Review, PLoS One, 4 (5) (2009), e5561.

[11] UNAIDS, Global Report: UNAIDS Report on the Global AIDS Epidemic 2013, World Health Organization, Geneva, Switzerland, 2013

[12] Implementing the WHO Stop TB Strategy: a handbook for national tuberculosis control programmes, Geneva, World Health Organization, 2008, p67.

[13] Global Tuberculosis Control 2017, WHO, Geneva, 2017.

[14] International Classification of Diseases (ICD), WHO, Geneva, 2010.

[15] A. Ahmad, M. Farman, F. Yasin, M. O. Ahmad, Dynamical transmission and effect of smoking in society, Int. J. Adv. Appl. Sci. 5 (2) (2018), 71-75.

[16] A. Ahmad, M. Farman, M.O. Ahmad, N. Raza, M. Abdullah, Dynamical behavior of SIR epidemic model with non-integer time fractional derivatives: A mathematical analysis, Int. J. Adv. Appl. Sci. 5 (1) (2018), 123-129.

[17] Farah Ashraf, Aqeel Ahmad, Muhammad Umer Saleem, Muhammad Farman, M. O. Ahmad, Dynamical behavior of HIV immunology model with non-integer time fractional derivatives, Int. J. Adv. Appl. Sci. 5(3) (2018), 39-45. 